\title{
An ecosystem for Linked Humanities Data
}

\author{
Rinke Hoekstra $^{1,5}$, Albert Meroño-Peñuela ${ }^{1,4}$, Kathrin Dentler ${ }^{1}$, Auke \\ Rijpma $^{2,7}$, Richard Zijdeman ${ }^{2,6}$, and Ivo Zandhuis ${ }^{3}$ \\ 1 Department of Computer Science, Vrije Universiteit Amsterdam, NL \\ \{rinke.hoekstra,albert.merono,k.dentler\}@vu.nl \\ 2 International Institute of Social History, KNAW, Amsterdam, NL \\ \{richard.zijdeman, auke.rijpma\}@iisg.nl \\ 3 Ivo Zandhuis Research \& Consultancy, Haarlem, NL ivo@zandhuis.nl \\ 4 Data Archiving and Networked Services, KNAW, The Hague, NL \\ ${ }^{5}$ Faculty of Law, University of Amsterdam, NL \\ ${ }^{6}$ University of Stirling, Stirling, UK \\ 7 Utrecht University, Utrecht, NL
}

\begin{abstract}
The main promise of the digital humanities is the ability to perform scholar studies at a much broader scale, and in a much more reusable fashion. The key enabler for such studies is the availability of sufficiently well described data. For the field of socio-economic history, data usually comes in a tabular form. Existing efforts to curate and publish datasets take a top-down approach and are focused on large collections. This paper presents QBer and the underlying structured data hub, which address the long tail of research data by catering for the needs of individual scholars. QBer allows researchers to publish their (small) datasets, link them to existing vocabularies and other datasets, and thereby contribute to a growing collection of interlinked datasets. We present QBer, and evaluate our first results by showing how our system facilitates two use cases in socio-economic history.
\end{abstract}

Keywords: Digital Humanities, Structured Data, Linked Data, QBer

\section{Introduction}

In a 2014 article in CACM, [10] describes digital humanities as a "movement and a push to apply the tools and methods of computing to the subject matter of the humanities." As the fuel of the computational method, the key enabler for digital humanities research is the availability of data in digital form. At the inauguration of the Center for Humanities and Technology (CHAT), José van Dijck, the president of the Dutch Royal Academy of Sciences, characterizes progress in this field as the growing ability to tremendously increase the scale at which humanities research takes place, thereby allowing for much broader views on the subject matter [25]. Tackling this important challenge for the digital humanities requires straightforward transposition of research queries from one humanities dataset to another, or even allow for direct cross-dataset querying. It is widely recognized that Linked Data technology is the most likely candidate 
to fill this gap. We argue that current efforts to increase the availability and accessibility of this data do not suffice. They do not cater for the "long tail of research data" [8], the large volumes of small datasets produced by individual researchers; and existing Linked Data tooling is too technology-oriented to be suitable for humanities researchers at large.

This paper presents QBer and the underlying CLARIAH Structured Data $\mathrm{Hub}(\mathrm{CSDH}),{ }^{8}$ whose aim is to address the limitations of current data-publishing practice in the digital humanities, and socio-economic history in particular. The $\mathrm{CSDH}$ integrates a selection of large datasets from this domain, while QBer is a user-facing web application that allows individual researchers to upload, convert and link 'clean' data to existing datasets and vocabularies in the hub without compromising the detail and heterogeneity of the original data (see Section 2). Under the hood, we convert all data to RDF, but QBer does not bother scholars with technical aspects. An inspector-view displays the result of the mappings - a growing network of interconnected datasets - in a visually appealing manner (See Figure 1). The visualization is just one of the incentives we are developing. The most important incentive will be the ability to allow for transposing research queries across datasets, and the ability to perform crossdataset querying. Section 4 describes two use-cases that evaluate the ability of QBer and the CSDH to fulfill that promise. We first discuss related work in Section 2 and describe the QBer and CSDH systems in Section 3.

\section{Related Work}

Historical data comprises text, audiovisual content or - in our case - data in the more traditional sense: structured data in tabular form. Preparing historical data for computational analysis takes considerable expertise and effort. As a result, digital data curation efforts are organized (and funded) in a top-down fashion, and focus on the enrichment of individual datasets and collections of sufficient importance and size. Examples are the North Atlantic Population Project (NAPP) [27], the Clio-Infra repository [5], and the Mosaic project. ${ }^{9}$ Such projects face three important issues. First, they often culminate in a website where subsets of the data can be downloaded, but cannot be programmatically accessed, isolating the data from efforts to cross-query over multiple datasets. Second, these projects enforce commitment to a shared standard: standardization leads to loss of detail, and thus information. The bigger a project is, the higher the cost of reconciling heterogeneity - in time, region, coding etc. - between the large number of sources involved. Finally, the scale of these projects is unsuited for the large volumes of important - but sometimes idiosyncratic - smaller datasets created by individual researchers: the long tail of research data [8].

For this last reason, it is difficult for individual researchers to make their data available in a sustainable way [28]. Despite evidence that sharing research data results in higher citation rates [24], researchers perceive little incentive to

\footnotetext{
${ }^{8} \mathrm{~A}$ screencast of the system is available at https://vimeo.com/158153564.

${ }^{9}$ See https://www.clio-infra.eu and http://www.censusmosaic.org/
} 
publish their data with sufficiently rich, machine interpretable metadata. Data publishing and archiving platforms such as EASY (in the Netherlands), ${ }^{10}$ Dataverse $^{11}$ or commercial platforms such as Figshare ${ }^{12}$ and Dryad ${ }^{13}$ aim to lower the threshold for data publishing and cater for increasing institutional pressure to archive research data. However, as argued in [13], the functionality of these platforms - data upload, data landing page, citable references, default licensing, long term preservation - is limited with respect to the types of provenance and content metadata that can be associated with publications, and they do not offer the flexibility of the Linked Data paradigm. This has a detrimental effect on both findability and reusability of research data.

In socio-economic history, a central challenge is to query data combined from multiple tabular sources: spreadsheets, databases and CSV files. The multiple benefits of Linked Data as a data integration method [11] encourage the representation of tabular sources as Linked Data. ${ }^{14}$ CSV and HTML tables can be represented in RDF using CSV2RDF and DRETa [16, 22]. For other tabular formats, like Microsoft Excel, Google Sheets, and tables encoded in JSON or XML, larger frameworks are needed, like Opencube [14], Grafter [26], and the combination of OpenRefine and DERI's RDF plugin [7,21]. TabLinker [19] uses a semi-automatic approach to represent multidimensional tables with missing observation/record data [1] as RDF Data Cube [6]. As in TopBraid Composer, ${ }^{15}$ TabLinker can use external mapping files instead of an interactive interface. These tools are targeted to relatively tech-savvy users for whom the conversion to RDF is a goal in itself. In our case, prospective users will benefit from interlinked data, but have no interest in the underlying technology.

An important question then is: how are these mapping files created? Work in ontology and vocabulary alignment, as in the OAEI, ${ }^{16}$ or identity reconciliation, aim to perform automatic alignments. Given the often very specific (historic) meaning of terms in our datasets, these techniques are likely to be error-prone, hard to optimize (given the heterogeneity of our data) and unacceptable to scholars. Interactive alignment tools, such as Amalgame [23], developed for the cultural heritage and digital humanities domains, are more promising, but treat the alignment task in isolation rather than as part of the data publishing process. Anzo for Excel ${ }^{17}$ is an extension for Microsoft Excel for mapping spreadsheet data to ontologies. Similarly, [2] and RightField ${ }^{18}$ allow for selecting terms from an ontology from within Excel spreadsheets, but these require the data to conform to a pre-defined template.

\footnotetext{
${ }^{10}$ See http://easy.dans.knaw.nl

${ }^{11}$ See http://dataverse.harvard.edu and http://dataverse.nl

${ }^{12}$ See http://figshare.com

${ }^{13}$ See http://datadryad.org

${ }^{14}$ For a comprehensive list, see e.g. https://github.com/timrdf/csv2rdf4lodautomation/wiki and http://www.w3.org/wiki/ConverterToRdf

${ }^{15}$ https://www.w3.org/2001/sw/wiki/TopBraid

${ }^{16}$ The Ontology Alignment Evaluation Initiative, see oaei.ontologymatching.org/

${ }^{17}$ https://www.w3.org/2001/sw/wiki/Anzo

${ }^{18}$ https://www.sysmo-db.org/rightfield
} 


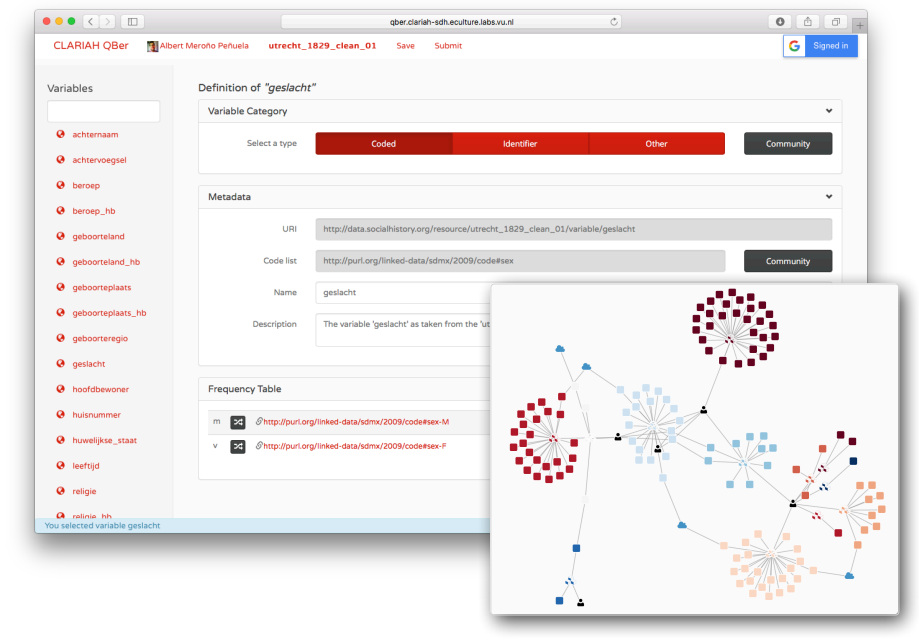

Fig. 1. Variable mapping screen of QBer with the variable 'geslacht' (sex) selected. The inspector can be seen in the bottom right.

\section{QBer and the Structured Data Hub}

To create a viable research ecosystem for Linked Humanites Data of all sizes, we need to combine expert knowledge with automated Linked Data generation. It should be easy and profitable for individual researchers to enrich and publish their data via our platform. To achieve the first goal, we developed QBer; ${ }^{19}$ an interactive tool that allows non-technical scholars to convert their data to RDF, to map the 'variables' (column names) and values in tabular files to Linked Data concept schemes, and to publish their data on the structured data hub. What sets QBer apart is that all Linked Data remains under the hood. To achieve the second goal, we build in direct feedback (reuse of existing content, visualizations, etc.) on top of the CSDH and demonstrate the research benefits of contributing data to it (see Section 4). We illustrate QBer by means of a walkthrough of the typical usage of the tool, and then summarize its connection with the CSDH.

Using QBer consists of interacting with three main views: the welcome screen, the mapping screen, and the inspector. In the welcome screen, users first authenticate with OAuth compatible services (e.g. Google accounts), and then select a raw dataset to work with. Datasets can be selected directly from the CSDH, or imported from a Dataverse collection by providing a DOI.

Once a dataset is loaded, QBer displays the mapping screen (Figure 1). This screen is divided into the variables sidebar (left) and the variable panel (right). The sidebar allows the user to search and select a variable (i.e. column) from the dataset. Once the user clicks on one variable, the variable panel will show that

\footnotetext{
${ }^{19}$ See https://github.com/CLARIAH/qber
} 
variable's details: the variable category, the variable metadata, and the value frequency table.

We distinguish between three variable categories: coded, identifier and other. Values for coded variables are mapped to corresponding concepts (skos:Concept) within a skos:ConceptScheme, which establishes all possible values the variable can take. If the variable is of type identifier, its values are mapped to dataset specific minted URIs. Finally, the values of variables of type other are mapped to literals instead of URIs. The 'Community' button gives access to all known predefined datacube dimensions. These come from LSD Dimensions, an index of dimensions used in Data Structure Definitions of RDF Data Cubes on the Web [18] and from datasets previously processed by QBer that now reside on the CSDH.

The variable metadata panel can be used to change the label and the description of the variable. If the variable has been specified to be "coded" in the previous pane, it can be linked to existing code lists curated by the Linked Open Data community. QBer provides access to all concept schemes from the Linked Data cache,${ }^{20}$ and the CSDH itself. If the variable is of type "other", this panel lets users define their own transformation function for literals.

The frequency table panel has three purposes. First, it allows for quick inspection of the distribution of all values of the selected variable, by displaying their frequency. Second, if the variable type is "coded", it lets the user map the default minted URI for the chosen value to any skos:Concept within the selected skos:ConceptScheme in the variable metadata panel. QBer also has a batch mapping mode that prompts the user to map all values of the variable interactively. Third, the panel shows the current mappings for values of the selected variable.

Mappings can be materialized in two ways. Users can click on Save in the navigation bar, which stores the current mapping status of all variables in their local cache. Clicking on Submit sends the mappings to the CSDH API, which integrates them with other datasets in the hub. Under the hood, the data is converted to a Nanopublication[9] with provenance metadata in PROV, where the assertion-graph is an RDF Data Cube representation of the data [6]. The RDF representation is a verbatim conversion of the data; mappings between the original values and pre-existing vocabularies are explicitly represented using SKOS mapping relations. This scheme allows for the co-existence of alternative interpretations (by different scholars) of the data, thus overcoming the standardization-limitation alluded to in Section 2.

The inspector, shown at the bottom right of Figure 1, allows users to explore the contents of the CSDH. The visualization shows a graph of nodes and edges, with different icons representing different node types. User nodes represent users that have submitted data to the hub, according to their provided OAuth identities in the welcome screen. Dataset nodes represent Data Struc-

\footnotetext{
${ }^{20}$ See http://lod.openlinksw.com we aim to extend this with schemes from the LODLaundromat, http://www.lodlaundromat.org.
} 
ture Definitions ${ }^{21}$ (DSD) submitted by these users. Dimension nodes represent dimensions (i.e. variables, columns in the raw data) within those DSD. Dimensions that are externally defined (e.g. by SDMX or some other external party) and are thus not directly used in datasets, are represented as cloud-icons. Users can interact with the inspector in two ways: hovering on nodes displays their properties; and dragging them moves the graph elements for better layout.

QBer and the Inspector work on top of the CSDH API, ${ }^{22}$ which carries out the backend functionality. This includes converting, storing, and managing POST and GET requests over datasets. The CSDH API functionality will be extended to cover standard data search, browsing and querying tasks. All data is currently stored in an OpenLink Virtuoso CE triplestore, ${ }^{23}$ but since CSDH communicates through the standard SPARQL protocols it is not tied to one triple store vendor.

\section{Evaluation}

In this section, we evaluate the results of our approach by means of two use cases in socio-economic history research. The first use case investigates the question as to whether the CSDH indeed allows research to be carried out on a broader scale. In this case, we transpose a query that was built to answer a research question aimed at a dataset of one country to a dataset that describes another country. The second use case investigates the question as to whether our system facilitates the workflow of a typical individual researcher.

\subsection{Use Case 1: Born Under a Bad Sign}

Economic and social history takes questions and methods from the social sciences to the historical record. An important line of research focuses on the determinants of historical inequality. One hypothesis here is that prenatal [3] and early-life conditions [12] have a strong impact on socioeconomic and health outcomes later in life. For example, a recent study on the United States found that people born in the years and states hit hardest during the Great Depression of the 1930s had lower incomes and higher work disability rates in 1970 and 1980 [29]. This study inspired this use case.

Most studies on the impact of early life conditions are case studies of single countries. Therefore, the extent to which results can be generalized - their external validity - is difficult to establish (e.g., differing impact of early life conditions in rich and poor countries). Moreover, historical data is often idiosyncratic. This

\footnotetext{
${ }^{21}$ According to [6], a Data Structure Definition "defines the structure of one or more datasets. In particular, it defines the dimensions, attributes and measures used in the dataset along with qualifying information such as ordering of dimensions and whether attributes are required or optiona".

${ }^{22}$ https://github.com/CLARIAH/wp4-csdh-api

${ }^{23}$ See http://www.openlinksw.com
} 
means that dataset-specific characteristics such as sampling and variable coding schemes might influence the results (see Section 2).

In this use case, we explore the relation between economic conditions in individuals' birth year and occupational status in the historical census records of Canada and Sweden in 1891. In many cases it would be necessary to link the two census datasets so that they can be queried in the same way. Here, however, we use two harmonized datasets from the North Atlantic Population Project (NAPP) [27]. Economic conditions are measured using historical GDP per capita figures from the Clio-Infra repository [5]. Because our outcome is occupational status, we have to enrich the occupations in the census with occupational codes and a status scheme. Because the NAPP-project uses an occupational classification that cannot provide internationally comparable occupational status scores, we have to map their occupational codes to the $\mathrm{HISCO}^{24}$ system, so that we can use the HISCAM cross-nationally comparable occupational status scheme [17, $15] .{ }^{25}$

In general terms, the data requirements are typical of recent trends in large database usage in economic and social history: 1) the primary unit of analysis is the individual (microdata); 2) a large number of observations is analyzed; 3 ) multiple micro-datasets are analyzed; 4) microlevel observations are linked to macro-level data through the dimensions time and geographical area; 5) qualitative data is encoded to extract more information from it.

Current Workflow. The traditional workflow to do this could include the following steps. First, the researcher has to find and download the datasets from multiple repositories. The datasets, which come in various formats, then have to be opened, and, if necessary, the variables have to be renamed, cleaned, and re-encoded to be able to join them with other datasets. We can rely on previous cleaning and harmonization efforts of the NAPP project, but in many other situations the researcher would have to do this manually. Finally, the joined data has to be saved in a format that can be used by a statistical program.

New Workflow. Using QBer and the CSDH, the workflow is as follows. Linkeddata tools are used to discover data on the hub. In our case, a linked data browser $^{26}$ and exploratory SPARQL queries were used. Note that to discover datasets and especially linked datasets on the CSDH, it is necessary that someone uploaded the datasets and created the links in the first place, for example by linking datasets to a common vocabulary. While it is unavoidable that someone has to do this at some point, the idea behind the hub is that if it is done once, the results can be re-used by other researchers.

Next, queries were built and stored on GitHub. The resultsets that these queries produce against the data hub are used to create the dataset that is to be analyzed. grlc, a tool we developed for creating Linked Data APIs using SPARQL queries in GitHub repositories, was helpful in exploring the data on

\footnotetext{
${ }^{24}$ HISCO: Historical International Standard Classification of Occupations.

${ }^{25}$ https://github.com/rlzijdeman/o-clack and http://www.camsis.stir.ac.uk/ hiscam/

${ }^{26}$ https://github.com/Data2Semantics/brwsr
} 


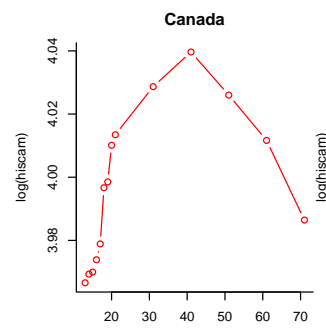

age

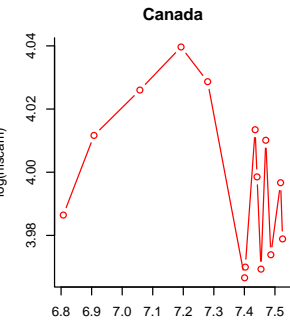

$\log (g d p p c)$

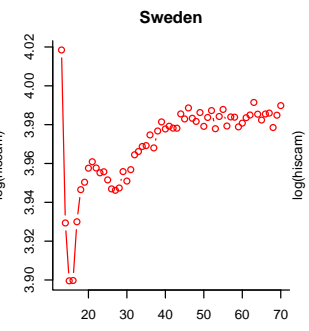

age

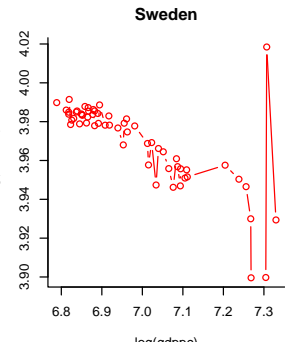

$\log (g d p p c)$

Fig. 2. HISCAM scores versus $\log ($ GDP per capita) in Canada (1891) and Sweden (1891

the hub and executing the eventual query $[20] \cdot{ }^{27}$ This tool can also be used to download the data directly into a statistical environment like $\mathrm{R}$ via HTTP requests, for example using curl. Alternatively, the CSDH can be queried directly from a statistical environment using SPARQL libraries.

Observations. While more sophisticated models are required to disentangle cohort, period and age effect [4], the results suggest that in Canada in 1891 the expected effects of early life-conditions are found: higher GDP per capita in a person's birth year was associated with higher occupational status at the time of the census. However, in Sweden, the opposite was the case (figure 2). This shows the relative ease at which the $\mathrm{CSDH}$ facilitates reusable research questions by means of query transposition.

\subsection{Use Case 2: Railway Strike}

The second use case takes the form of a user study. It is about the "Dwarsliggers" 28 dataset by Ivo Zandhuis that collects data pertaining to a solidarity strike at the maintenance workshop of the Holland Railway Company (Hollandsche IJzeren Spoorweg-Maatschappij), in the Dutch city of Haarlem in 1903. From a sociological perspective, strikes are of interest for research on social cohesion as it deals both with the question of when and why people live peaceful together (even when in disagreement) and the question of how collective action is successfully organized, a prerequisite for a successful strike. The Dwarsliggers dataset is one of the few historical cases where data on strike behavior is available at the individual level.

The creation and use of this dataset is exemplary of the workflow of small to medium quantitative historical research projects in the sense that it relies on multiple data sources that need to be connected in order to answer the research questions. We briefly discuss this workflow, and then show the impact that QBer and the CSDH have.

\footnotetext{
${ }^{27}$ https://github.com/CLARIAH/grlc

${ }^{28}$ In Dutch, a "dwarsligger" can mean both a railroad tie, and an obstructive person.
} 
Current Workflow. Zandhuis' current workflow is very similar to the one reported in the first use case. He first digitized the main dataset on the strike behaviour of employees at the maintenance workshop of the railway company $(N=1163)$. Next, he gathered data from multiple sources in which these employees also appear, adding individual characteristics that explain strike behaviour. For example, he derived family situations from the Dutch civil registers, and the economic position from tax registers, resulting in a separate dataset per source. Next, he inserted these datasets into a SQL database. In order to derive a concise subset to analyze his research questions, using e.g. QGIS, Gephi or R, he wrote SQL queries to extract the relevant information. These queries are usually added as an appendix to his research papers.

New Workflow. In collaboration with Zandhuis, we revisited this workflow using QBer. Zandhuis, as most historians, uses spreadsheets to enter data, and uses a specific layout to enhance the speed and quality of data entry. The first step was to convert the data to a collection of .csv files. This is just a temporary limitation, as the CSDH is not necessarily restricted to CSV files. It uses the Python Pandas library ${ }^{29}$ for loading tabular files into a data frame.

The second step involves visiting each data file in turn, and linking the data to vocabularies and through them to other datasources. Data about the past often comes with a wide variety of potential values for a single variable. Religion, for example, can have dozens of different labels as new religions came about and old religions disappeared. As described in Section 3, QBer provides access to a large range of such classifications, basically all those available in the Linked Data cloud and the CSDH. For example, QBer provides all occupation concepts from the HISCO classification used in the first use case [17]. Researchers can use occupational labels to get the correct codes from the latest version of this classification and, eventually, concepts linked to it. QBer however also shows the results of earlier coding efforts, so that historians can benefit from these (e.g. another dataset may have the same literal value already mapped to as HISCO code). This step is new compared to Zandhuis' original workflow. The linking of occupational labels now enables him to combine an employee with his social status (HISCAM). This allows him to directly include a new, relevant, aspect in his study. Moreover, since QBer makes coding decisions explicit, they can be made subject to the same peer review procedure used to assess the quality of a research paper. In the CSDH, original values of the dataset and the mapped codings (potentially by different researchers) live side-by-side. Thus QBer adds to the ease of use in coding variables, increases flexibility by allowing for multiple interpretations, and allows for more rigorous evaluation of coding efforts. The inspector graph of Figure 1 depicts the result of the new workflow.

The third step was then to query the datasets in order to retrieve the subset of data needed for analysis. As in the first use case, we design SPARQL queries that, when stored on GitHub, can be directly executed through the grlc API. This makes replication of research much easier: rather than including the query as an appendix of a research paper, the query is now a first order citizen and can

\footnotetext{
${ }^{29}$ See http://pandas.pydata.org
} 
even be applied to other datasets that use the same mappings. Again, through the API, these queries can easily be accessed from within R, in order to perform statistical analysis. Indeed, the grlc API is convenient, but it is a lot to ask non-computer science researchers to design SPARQL queries. However, as we progress, we expect to be able to identify a collection of standard SPARQL query templates that we can expose in this manner (see also [20]).

To illustrate this, consider that since the Dwarsliggers collection contains multiple datasets on the same individuals at the same point in time, there are multiple observations of the same characteristics (e.g. age, gender, occupation, religion). However, the sources differ in accuracy. For example, measuring marital status is one of the key aims of the civil registry, while personnel files may contain information on marital status, but it is not of a key concern for a company to get this measurement right. By having all datasets mapped to vocabularies through QBer and having the queries stored in GitHub and executed by grlc, each query can readily be repeated using different sources on the same variables. This is useful as a robustness check of the analysis or even be used in what historians refer to as a 'source criticism' (a reflection of the quality and usefulness of a source). This, again, is similar to the first use case, but it emphasizes an additional role for the queries as so-called 'edit rules'.

Observations. To conclude, this use case shows that the QBer tool and related infrastructure provides detailed insight in how the data is organized, linked and analyzed. Furthermore, the data can be queried live. This ensures reusable research activities; not just reusable data.

\section{Conclusion}

The preceding sections presented QBer and the CSDH to address the limitations of existing digital humanities data curation projects in facilitating 1) the long tail of research data and 2) research at a broader scale, enabling cross-dataset querying and reuse of queries. We argued that existing Linked Data publishing and mapping tools do not meet the needs of scholars that are not technologically versed (or interested).

QBer and the CSDH enable individual scholars to publish and use their data in a flexible manner. QBer allows researchers to publish their (small) datasets, link them to existing vocabularies and other datasets, and thereby contribute to a growing collection of interlinked datasets hosted by the CSDH. The CSDH offers services for inspecting data, and (in combination with grlc) reusable querying across multiple datasets. We illustrated these features by means of two use cases. The first shows the ability of the Linked Data paradigm used in the CSDH to significantly lower the effort needed to do comparative research (even when the data was published as part of the same larger standardization effort). The second use case shows how publishing data through QBer allows individual researchers to have more grip on their data, to be more explicit regarding data interpretation (coding) and, via the CSDH, to be able to answer more questions for free (e.g. the mapping through HISCO to HISCAM). 
Of course, there still is room for expansion. To ensure uniqueness of identifiers, historical 'codes' need to be mapped to URIs. This is technically trivial, but historians are not used to these lengthy identifiers in their statistical analyses. Secondly, formulating research questions as queries requires an understanding of the structure of the data. Given the large numbers of triples involved, this can be difficult. As said above, standard APIs based on SPARQL query templates should solve some of this problem, but offering a user-friendly data inspection tool is high on our list. SPARQL templates allow us to solve another issue: allowing for free-form querying can have a detrimental effect on the performance of the CSDH. The use of templates enables more efficient use of caching strategies.

But even without such improvements, we believe that the use cases show that QBer and the CSDH already broaden the scope of supported workflows and data in our ecosystem, and bring the benefits of Linked Data and the Semantic Web at the fingertips of humanities scholars.

Acknowledgements This work was funded by the CLARIAH project of the Dutch Science Foundation (NWO) and the Dutch national programme COMMIT.

\section{References}

1. Ashkpour, A., Meroño-Peñuela, A., Mandemakers, K.: The Dutch historical censuses: Harmonization and RDF. Historical Methods: A Journal of Quantitative and Interdisciplinary History 48 (2015)

2. van Assem, M., Rijgersberg, H., Wigham, M., Top, J.: Converting and annotating quantitative data tables. In: Proceedings of the International Semantic Web Conference (ISWC 2010). LNCS, vol. 6496, pp. 16-31. Springer (2010)

3. Barker, D.J.: The fetal and infant origins of adult disease. BMJ: British Medical Journal 301(6761), 1111 (1990)

4. Bartels, L.M., Jackman, S.: A generational model of political learning. Electoral Studies 33, 7-18 (2014)

5. Bolt, J., Timmer, M., van Zanden, J.L.: GDP per capita since 1820. In: How Was Life? Global well-being since 1820, pp. 57-72. Organisation for Economic Co-operation and Development (Oct 2014)

6. Cyganiak, R., Reynolds, D., Tennison, J.: The RDF Data Cube Vocabulary. Tech. rep., W3C (2013), http://www.w3.org/TR/vocab-data-cube/

7. DERI: RDF Refine - a Google Refine extension for exporting RDF. Tech. rep., Digital Enterprise Research Institute (2015), http://refine.deri.ie/

8. Ferguson, A.R., Nielson, J.L., Cragin, M.H., Bandrowski, A.E., Martone, M.E.: Big data from small data: data-sharing in the 'long tail' of neuroscience. Nature neuroscience 17(11), 1442-1447 (2014)

9. Groth, P., Gibson, A., Velterop, J.: The anatomy of a nanopublication. Information Services and Use 30(1-2), 51-56 (2010)

10. Haigh, T.: We have never been digital. Commun. ACM 57(9), 24-28 (Sep 2014)

11. Heath, T., Bizer, C.: Linked Data: Evolving the Web into a Global Data Space. Morgan and Claypool, 1st edn. (2011)

12. Heckman, J.J.: Skill formation and the economics of investing in disadvantaged children. Science 312(5782), 1900-1902 (Jun 2006), http://www.sciencemag.org/ content $/ 312 / 5782 / 1900$ 
13. Hoekstra, R., Groth, P.: Linkitup: Link discovery for research data. AAAI Fall Symposium Series Technical Reports (FS-13-01), 28-35 (2013)

14. Kalampokis, E., Nikolov, A., et al.: Exploiting Linked Data Cubes with OpenCube Toolkit. In: Posters and Demos Track, 13th International Semantic Web Conference (ISWC2014). vol. 1272. CEUR-WS, Riva del Garda, Italy (2014), http://ceurws.org/Vol-1272/paper_109.pdf

15. Lambert, P.S., Zijdeman, R.L., Van Leeuwen, M.H., Maas, I., Prandy, K.: The construction of HISCAM: A stratification scale based on social interactions for historical comparative research. Historical Methods: A Journal of Quantitative and Interdisciplinary History 46(2), 77-89 (2013)

16. Lebo, T., McCusker, J.: csv2rdf4lod. Tech. rep., Tetherless World, RPI (2012), https://github.com/timrdf/csv2rdf4lod-automation/wiki

17. van Leeuwen, M., Maas, I., Miles, A.: HISCO: Historical International Standard Classification of Occupations. Leuven University Press (2002)

18. Meroño-Peñuela, A.: LSD dimensions: Use and reuse of linked statistical data. In: Knowledge Engineering and Knowledge Management (EKAW 2014). LNCS, vol. 8982, pp. 159-163 (2014)

19. Meroño-Peñuela, A., Ashkpour, A., Rietveld, L., Hoekstra, R., Schlobach, S.: Linked Humanities Data: The next frontier? In: 2nd International Workshop on Linked Science (LISC2012), ISWC. vol. 951. CEUR-WS (2012), http://ceurws.org/Vol-951/

20. Meroño-Peñuela, A., Hoekstra, R.: Using grlc to spice up GitHub repostories as Linked Data APIs. In: Proceedings of the Services and Applications over Linked APIs and Data workshop, ESWC 2016 (2016), to appear

21. Morris, T., Guidry, T., Magdinie, M.: OpenRefine: A free, open source, powerful tool for working with messy data. Tech. rep., The OpenRefine Development Team (2015), http://openrefine.org/

22. Muñoz, E., Hogan, A., Mileo, A.: DRETa: Extracting RDF from Wikitables. In: Int. Semantic Web Conference, posters and demos. pp. 98-92. CEUR-WS (2013)

23. van Ossenbruggen, J., Hildebrand, M., de Boer, V.: Interactive vocabulary alignment. In: Research and Advanced Technology for Digital Libraries (TPDL 2011). LNCS, vol. 6966, pp. 296-307. Springer-Verlag, Berlin, Heidelberg (2011)

24. Piwowar, H.A., Day, R.S., Fridsma, D.B.: Sharing detailed research data is associated with increased citation rate. PloS one 2(3), e308 (Jan 2007), http: //dx.plos.org/10.1371/journal.pone.0000308

25. Renckens, E.: Digital humanities verfrissen onze blik op bestaande data. E-Data \& Research 10 (February 2016)

26. Roman, D., Nikolov, N., et al.: DataGraft: One-stop-shop for Open Data management. Semantic Web - Interoperability, Usability, Applicability (2016), under review, http://www.semantic-web-journal.net/content/datagraft-one-stopshop-open-data-management

27. Ruggles, S., Roberts, E., Sarkar, S., Sobek, M.: The North Atlantic Population project: Progress and prospects. Historical Methods: A Journal of Quantitative and Interdisciplinary History 44(1), 1-6 (Jan 2011)

28. Tenopir, C., Allard, S., Douglass, K., Aydinoglu, A.U., Wu, L., Read, E., Manoff, M., Frame, M.: Data sharing by scientists: Practices and perceptions. PLoS ONE 6(6), e21101 (06 2011), http://dx.doi.org/10.1371\%2Fjournal.pone.0021101

29. Thomasson, M.A., Fishback, P.V.: Hard times in the land of plenty: The effect on income and disability later in life for people born during the great depression. Explorations in Economic History 54, 64-78 (Oct 2014) 